\title{
Exportación de Objetos de Arte, Editorial e Industria Gráfica: Perspectiva para Argentina y Chile ${ }^{* *}$
}

\author{
Bárbara Valenzuela-Klagges ${ }^{1 *}$, José Meza-Guzmán ${ }^{1}$, Darcy Fuenzalida-O’Shee ${ }^{2}$, Iván Valenzuela-Klagges ${ }^{3}$
}

Resumen: Frente a la oportunidad que brinda el mercado de bienes creativos a la diversificación exportadora y a la creación de empleo e inclusión, el objetivo principal de este artículo es analizar el mercado mundial y regional de objetos de arte, colección, antigüedad, editorial e industrias gráficas y determinar las variables que pueden estar influyendo en el comportamiento exportador de estos bienes creativos en Argentina y Chile durante el período 2002-2015. Para ello, se desarrollará una investigación descriptiva y empírica, revisando detalladamente datos y evidencias y; posteriormente, se determinará un modelo gravitacional ampliado que será estimado mediante datos de panel estáticos y dinámicos. Los principales resultados indican que el comportamiento exportador de estos bienes creativos en Argentina y Chile está explicado por un modelo dinámico, en que a mayor producto nacional bruto del país importador y mejor facilidad reglamentaria y legal para hacer negocios mayores será la exportación de estos bienes.

Palabra Clave: arte y colección; objeto de antigüedad; industria gráfica; editorial; exportación; bienes creativos.

\section{Export of Art, Editorial and Graphic Objects: Perspective for Argentina and Chile}

Abstract: The main objective of this article is to analyze the world and regional market for objects of art, collection, antiquity, publishing and graphic industries, and in order to analyze the opportunities offered by the creative goods market to export diversification and job creation and inclusion. Determine the variables that may be influencing the export behavior of these creative goods in Argentina and Chile during the period 2002-2015. For this, a descriptive and empirical investigation will be developed, reviewing in detail data and evidences and; Later, an extended gravitational model will be determined which will be estimated using static and dynamic panel data. The main results indicate that the export behavior of these creative goods in Argentina and Chile is explained by a dynamic model in which the greater gross national product of the importing country and better regulatory and legal facility to do business will be the export of these goods.

Keyword: art and collection; object of seniority; graphic industry; editorial; export; creative goods.

\section{Introducción}

Dada la desaceleración de la economía mundial, según CEPAL (2014), es necesario dinamizar el crecimiento económico de América Latina implicando amplios desafíos para la región, debido a que el aumento de la demanda externa será lento y complejo y dificultará el aporte que las exportaciones puedan hacer al crecimiento. Si bien el comercio internacional sigue siendo una fuente de oportunidades, CEPAL (2014) sugiere que Latinoamérica y el Caribe deben redoblar sus esfuerzos por volverse más competitivos en sus mercados externos y, a la vez, incrementar los esfuerzos por abrir nuevos mercados y diversificar sus exportaciones. Adicionalmente, otros autores como Beltrán (2013), Ocampo (2015) y Romero (2016) reconocen el lento dinamismo previsible del comercio internacional latinoamericano y la creciente dependencia de América Latina de sus exportaciones en recursos naturales, que hace aún más necesario repensar la tarea de mejorar la competitividad y la calidad de la canasta exportadora, así como el balance entre el mercado interno y el externo.

Ante esta necesidad de diversificar la canasta exportadora y abrir nuevos mercados, las industrias culturales y creativas podrían establecerse como un aporte importante para el crecimiento económico de países de América Latina. Al respecto, Barciela, López y Melgarejo (2012) señalan que la cultura transforma las realidades en "espacios de la innovación» culturalmente dinámicos y activos, internacionales por vocación, capaces de ofrecer a sus residentes la posibilidad de experiencias estimulantes, motivadoras en la inversión de nuevas capacidades, constituyéndose las industrias creativas en un aporte al desarrollo sostenible.

Según el informe de EY (2015) para el año 2013, este tipo de industrias generaron un ingreso mundial sobre los 2,25 billones de dólares y dieron empleo a 29 millones de personas, siendo la televisión, artes visuales y periódicos y revistas los principales mercados creativos. En el mismo año, Latinoamérica generaba ingresos por 124.000 millones de dólares (el 6\% del mercado mundial) y 1,9 millones de empleos sólo por el desarrollo de dicha industria creativa, sustentado en los siguientes activos creativos: 131 sitios inscritos como patrimonio mundial que se contabilizaron como activos creativos, el dinamismo de escritores latinoamericanos y los conglomerados de multimedia en radios, prensa y televisión (EY, 2015). Al estudiar las industrias culturales en Cali-Colombia, Alonso y Gallego (2011) observaron que, para los años 2005 y 2008, este tipo de industria aportó $1,01 \%$ y $1,22 \%$, respectivamente, al producto interno bruto (PIB) municipal;

(1) Departamento de Industria y Economía. Facultad de Ingeniería. Universidad de Playa Ancha, Valparaíso-Chile.

(2) Departamento de Ingeniería Comercial, Universidad Técnica Federico Santa María, Valparaíso-Chile.

(3) Universidad Católica de Valparaíso y Universidad de Playa Ancha

${ }^{*}$ Corresponding author: barbara.valenzuela@upla.cl

** Artículo ganador de la selección de Emprendimiento e Innovación del Encuentro Nacional de Facultades de Administración y Economía, ENEFA 2017. 
generó 6.632 empleos en el 2005, representando el 2,7\% del empleo de la ciudad en ese año, y; las actividades que más aportaron a las ventas fueron fotografía $(51,5 \%)$ y comercio especializado al por menor de libros (18,6\%).

Con el propósito de contribuir a la diversificación de la exportación con políticas adaptadas a la realidad cultural de Argentina y Chile y como una forma de impulsar la cultura con orientaciones económicas y creativas en estos dos países, surgen las preguntas de investigación que motivan el desarrollo de este estudio. Las preguntas centrales de esta investigación son: ¿Qué características y evolución presenta el mercado mundial de objetos de arte, colección, antigüedad, editorial e industrias gráficas y cuál es la participación de Chile y Argentina?; ¿Qué factores están influyendo en el comportamiento exportador de estos bienes creativos en Chile y Argentina? y; ¿Que políticas culturales y comerciales pueden incentivar este tipo de mercado en estos países?

Por consiguiente, el objetivo principal de este artículo es analizar el mercado mundial y regional de objetos de arte, colección, antigüedad, editorial e industrias gráficas y determinar las variables que pueden estar influyendo en el comportamiento exportador de estos bienes creativos en Argentina y Chile durante el período 2002-2015. El estudio se centra en estos dos países sudamericanos, en primer lugar, por presentar continuidad exportadora durante todo el período a 37 países de destino, permitiendo construir una muestra de 1008 observaciones en total. En segundo lugar, por presentar semejanza idiomática, lo que permite medir el efecto de un idioma diferente del país importador; porque estos dos países tienen salida a diferentes océanos, por lo que aspectos logísticos, de costos, ubicación geográfica y facilitación comercial pueden ser factores influyentes para la exportación de estos bienes.

En la metodología aplicada en este estudio se consideró el planteamiento de algunos estudios, como el de Urrea (2012) el cual plantea que el mercado del arte y su comercialización está vigente en varios países, pero que es difícil la labor de investigación por la falta de información en los registros, por los constantes cambios de partidas o divisiones arancelarias y por las características de desarrollo del mercado en particular. De este modo, el desarrollo metodológico de esta investigación ha centrado sus esfuerzos en la búsqueda de datos, revisando cuidadosamente la información expuesta por la fuente TradeMap (2015), ALADI (2016) y de las aduanas de cada país en estudio. Dado que una de las problemáticas de investigar los bienes creativos es la falta generalizada de recursos y experiencia para asegurar la alta calidad en labores estadísticas sobre el sector creativo (Van der Pol, 2007), se contrastaron las fuentes y se verificaron los datos por país, año y socio comercial. Aunque no había una variación significativa del monto exportado en estas partidas arancelarias, se observa que la base Trade-Map y ALADI solo contabilizaban datos mayor a 1000 USD, por lo que los registros de las aduanas se utilizaron para verificar montos menores y completar la muestra.
Revisando detalladamente datos y evidencias, se desarrollará una investigación descriptiva y empírica y, posteriormente, se determinará un modelo gravitacional ampliado que será estimado mediante datos de panel estáticos con efectos fijos y aleatorios y dinámicos, incluyendo en la muestra a 37 socios comerciales de Chile y Argentina durante el período 2002-2015, lo que representa el 96\% del total exportado promedio de objetos de arte, colección, antigüedad, editorial e industrias gráficas durante este periodo.

Este estudio busca aportar al debate científico y cultural, ya que no se registran estudios con este objetivo y metodología en Argentina y Chile, como tampoco, se observan estudios semejantes en Sudamérica.

\section{Demanda Mundial y Comportamiento Exportador Sudamericano}

Los objetos de artes, colección y antigüedad y editoriales e industrias gráficas son clasificados como bienes culturales y creativos, según definición de UNESCO (2016) que define a las industrias culturales y creativas como aquellos sectores de actividad organizada que tienen como objeto la producción o la reproducción, la promoción, la difusión y/o la comercialización de bienes, servicios y actividades de contenido cultural, artístico o patrimonial, incluyendo, también, actividades relacionadas como la publicidad y el diseño gráfico, que contribuyen decisivamente en este proceso. A diferencia de un mercado de bienes tradicionales de producción y exportaciones de Argentina y Chile como el cobre y sus derivados, soya y otros granos, forestal y minerales, en general; este tipo de mercado cultural y creativo es conformado principalmente por las casas de subastas, grupo de anticuarios, clientes internacionales de trato directo, coleccionistas y artesanos, destacando que algunas de estas transacciones son informales y no se registran como bienes exportados. Adicionalmente, presenta características y evidencias de un mercado de competencia imperfecta, con un desarrollo exponencial en los últimos años, que ha dificultado tanto a la valorización de estos bienes y su gestión aduanera, al registro del derecho de autor y su control como a las políticas públicas y sus propuestas de presupuesto orientado a esta industria. Rowan (2014) reconoce que se vive un "inusitado momento de transición en lo que a las políticas culturales se refiere" (pp.1). Para el autor, se encuentran agotadas las dos principales tradiciones sobre las que se sustentaban las políticas culturales: aquella tradición en que la cultura es percibida como un ente educador y aquella tradición más reciente en que describe la cultura como un elemento de desarrollo y crecimiento económico. En el nuevo modelo y en actual transición, el principal gestor comercial es el artista, programador, diseñador o músico. No obstante, últimamente se ha perseverado en la construcción de una institucionalidad compuesta de incubadoras público-privadas, planes de promoción, oficinas de información, eventos, charlas y talleres, líneas de financiación o espacios de coworking, complementada con programas de televisión, eventos públicos, películas, libros y revistas, Rowan (2014) enfatiza las críticas y protestas que se han ido acumulando contra este tipo de iniciativas, cuyas estimaciones económicas están en entredicho. 


\subsection{Mercado de Arte, Colección y Antigüedad (Partida 97)}

Para Jarque (2007) el mercado del arte en los últimos veinte años es imparable, producto de nuevas tendencias marcadas por la educación y el desarrollo económico, lo que conllevará a una nueva relación entre los componentes del mercado del arte y los coleccionistas o instituciones que amplían sus colecciones. En la actualidad y ante un mercado del arte más dinámico, fraguándose en medio de los países productores, surgen las tendencias de una disciplina sobre otra en que la evolución de los artistas y la valorización de sus obras como piezas de valor no se pueden regir por la oferta y la demanda. Las potencias mundiales, como Inglaterra, Estados Unidos y China, han ingresados fuertemente a competir en dicho mercado. Inglaterra posee las subastas de mayor reconocimiento internacional (Sotheby's y Christie's) y Estados Unidos ostenta la capital del arte mundial, Nueva York, caracterizado por eventos artísticos de excelencia y subastas de prestigio mundial. China ha tomado protagonismo en el mercado del arte, ocupando el lugar de Francia en el arte contemporáneo y concentrando su fuerza en las ventas de arte moderno, participación que va en aumento.

Según Pérez y Sánchez (2011), las subastas constituyen uno de los intermediarios principales del mercado del arte y de los coleccionables, siendo su funcionamiento fácil, accesible y sobre todo ampliamente difundido. A nivel internacional, "Sotheby's y Christie's son actualmente las entidades más carismáticas y reconocidas de este peculiar mercado" (pp.543), abarcando el 76\% del mercado de obras de arte. Adicionalmente, los autores destacan el "boom" de subastas por internet "...que permite al mediano y pequeño coleccionista facilitar el acceso a este mercado." (pp.544). Además, algunas subastas de reconocido prestigio utilizan su reputación como garantes para ofertar piezas de bajo costo, a través de salas virtuales como Ebay.es o Mercadolibre.com, y logran ampliar su mercado con clientes y medios de distribución.

En la Figura 1, se puede observar los países importadores de objetos de artes, de colección o de antigüedad en el 2015. Después del desplome del crecimiento económico y del comercio de bienes y servicios a nivel mundial en el 2009, se observa una pronta recuperación en el 2010, registrándose un aumento del 14,5\% de las exportaciones de mercadería y una producción mundial de un 3,6\% del PIB (Wto, 2011). Sin embargo, el mercado mundial de objetos de arte, colección y antigüedad comienza a recuperarse más lentamente que el general, observándose un repunte recién en el 2011. Estados Unidos, Reino Unido y Suiza son los principales países demandantes internacionales de estos bienes con un 68,4\% del mercado en el 2014 (Figura 1).

Figura 1. Países Importadores de Objetos de Arte, Colección y Antigüedad en el 2015

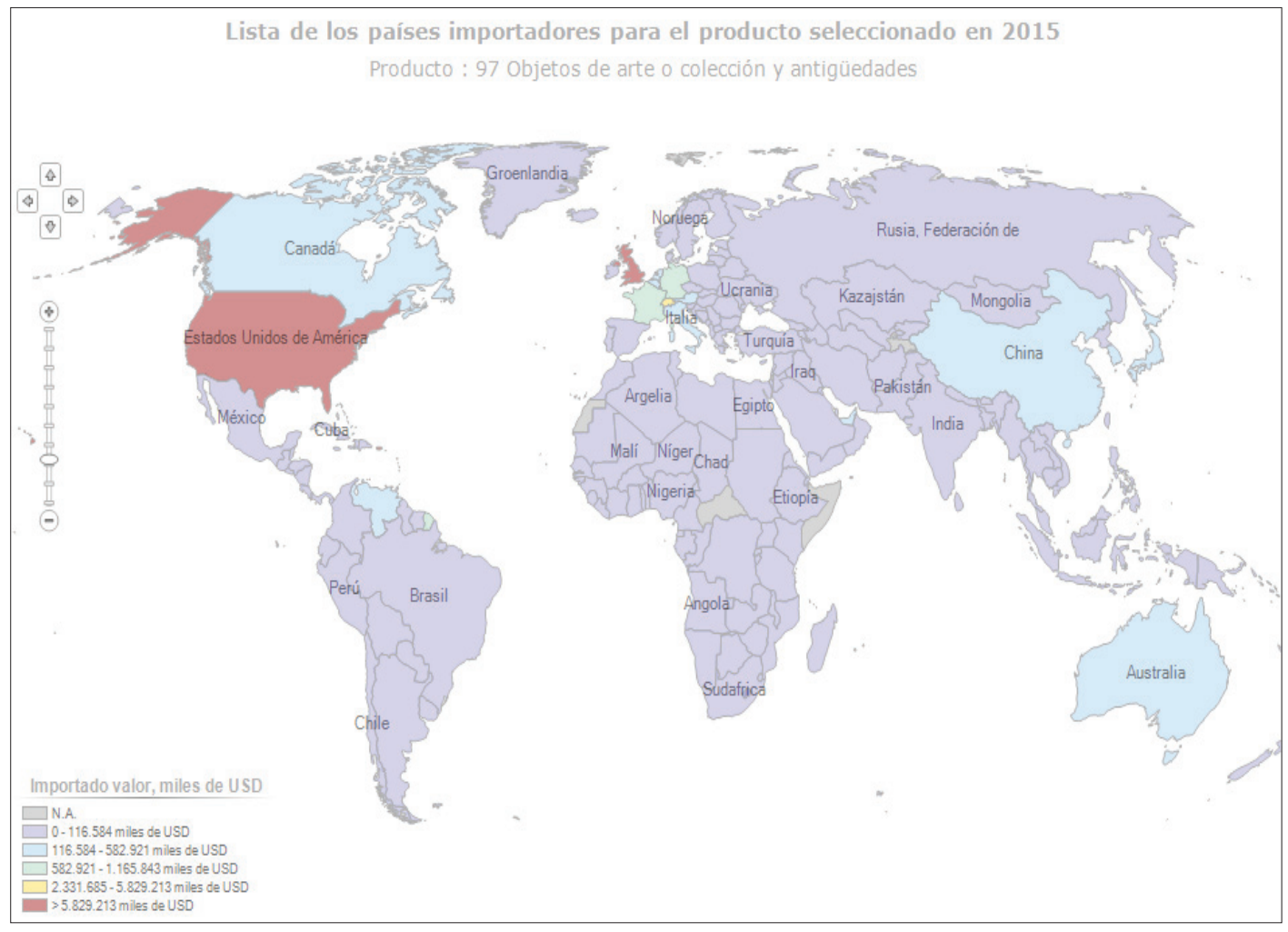

Fuente: Cálculo de CCI basado en estadísticos de UM COMTRADE y Trade-Map (2015). 
La evolución exportadora sudamericana de estos bienes se manifiesta en el promedio de crecimiento anual del $110 \%$, superior al dinamismo observado a nivel mundial. Sin embargo, el monto exportado es insignificante, no superior al $0,36 \%$ de las exportaciones totales. Los principales exportadores sudamericanos son Brasil, Venezuela, Colombia y Argentina (ALADI, 2017). En la Tabla 1, se observa la baja considerable del monto exportado de bienes de arte, colección y antigüedad en Argentina, principalmente en pinturas, dibujos y artículos decorados a mano; obras originales de escultura y antigüedades de más de 100 años. En la Tabla 2, se observa en Chile un aumento paulatino del monto exportado de estos bienes, duplicando sus exportaciones en el 2015 (respecto del 2012) en pinturas, dibujos y artículos decorados a mano y aumenta progresivamente su exportación en

Tabla 1. Exportación de Argentina Partida 97, Valores en Miles de USD (2012-2015)

\begin{tabular}{|c|c|c|c|c|}
\hline Descripción & 2012 & 2013 & 2014 & 2015 \\
\hline $\begin{array}{l}\text { Pinturas y dibujos, hechos total- } \\
\text { mente a mano, excepto los dibujos } \\
\text { de la partida } 49.06 \text { y los artículos } \\
\text { manufacturados decorados a mano; } \\
\text { collages y cuadros similares. }\end{array}$ & 3.486 & 2.576 & 922 & 858 \\
\hline $\begin{array}{l}\text { Grabados, estampas y litografías } \\
\text { originales. }\end{array}$ & 4 & 21 & 0 & 50 \\
\hline $\begin{array}{l}\text { Obras originales de estatuaria o es- } \\
\text { cultura, de cualquier materia. }\end{array}$ & 840 & 570 & 525 & 362 \\
\hline $\begin{array}{l}\text { Sellos de correo, timbres fiscales, } \\
\text { marcas postales, sobres primer día, } \\
\text { enteros postales, demás artículos } \\
\text { franqueados y análogos. }\end{array}$ & & & 5 & \\
\hline $\begin{array}{l}\text { Colecciones y especímenes para } \\
\text { colecciones de zoología, botánica, } \\
\text { mineralogía o anatomía o que ten- } \\
\text { gan interés histórico, arqueológico, } \\
\text { paleontológico, etnográfico o nu- } \\
\text { mismático. }\end{array}$ & 261 & 348 & 369 & 290 \\
\hline Antigüedades de más de cien años. & 627 & 62 & 535 & 223 \\
\hline Total & 5.218 & 3.577 & 2.356 & 1.783 \\
\hline
\end{tabular}

Fuente: Elaboración propia, según datos ALADI (2017). colección y especímenes de interés histórico, arqueológico, paleontológico, etnográfico o numismático. Las exportaciones de sellos de correo, timbres, postales y sobres de colección son casi nulas en estos dos países.

\subsection{Productos Editoriales, Prensa e Industrias Gráficas (Partida 49)}

En la Figura 2, se puede observar los países importadores de productos editoriales, prensa e industria gráfica en el 2015. Desde el 2004 al 2015, la importación de estos productos creativos ha aumentado en un 25\%, alcanzando un máximo en el 2008 de 46.185 millones de USD y, afectada por la crisis subprime, disminuye en el 2009 con una recuperación paulatina a contar del 2010. En el 2015, este tipo de importación alcanza los 42.427 millones de USD.

Tabla 2. Exportación de Chile Partida 97, Valores en Miles de USD (2012-2015)

\begin{tabular}{|l|r|r|r|r|}
\hline Descripción & 2012 & 2013 & 2014 & 2015 \\
\hline $\begin{array}{l}\text { Pinturas y dibujos, hechos totalmente } \\
\text { a mano, excepto los dibujos de la par- } \\
\text { tida 49.06 y los artículos manufactur- } \\
\text { ados decorados a mano; collages y } \\
\text { cuadros similares. }\end{array}$ & 138 & 135 & 153 & 301 \\
\hline $\begin{array}{l}\text { Grabados, estampas y litografías orig- } \\
\text { inales. }\end{array}$ & 0 & 34 & 0 & 23 \\
\hline $\begin{array}{l}\text { Obras originales de estatuaria o escul- } \\
\text { tura, de cualquier materia. }\end{array}$ & 122 & 322 & 4 & 28 \\
\hline $\begin{array}{l}\text { Sellos de correo, timbres fiscales, } \\
\text { marcas postales, sobres primer día, } \\
\text { enteros postales, demás artículos } \\
\text { franqueados y análogos. }\end{array}$ & 54 & 1 & & \\
\hline $\begin{array}{l}\text { Colecciones y especímenes para col- } \\
\text { ecciones de zoología, botánica, min- } \\
\text { eralogía o anatomía o que tengan } \\
\text { interés histórico, arqueológico, pale- } \\
\text { ontológico, etnográfico o numismáti- } \\
\text { co. }\end{array}$ & 51 & 5 & 368 & 322 \\
\hline Antigüedades de más de cien años. & 41 & 139 & 0 & 50 \\
\hline Total & 353 & 689 & 525 & 724 \\
\hline
\end{tabular}

Fuente: Elaboración propia, según datos ALADI (2017).

Los principales países importadores son Francia (13\%), Estados Unidos (10\%) y Reino Unido (6\%) del total importado a nivel mundial.

Al analizar las exportaciones mundiales, Francia, Estados Unidos, Alemania, Reino Unido y China son los principales exportadores de productos editoriales, prensa e industria gráfica. Mientras que, la tendencia exportadora en Sudamérica va progresivamente en disminución con el 0,4\% del mercado mundial en el 2015. Los principales países exportadores son Perú, Argentina, Brasil y Chile. 
Figura 2. Países Importadores de Productos Editoriales, Prensa y Gráfica en el 2015

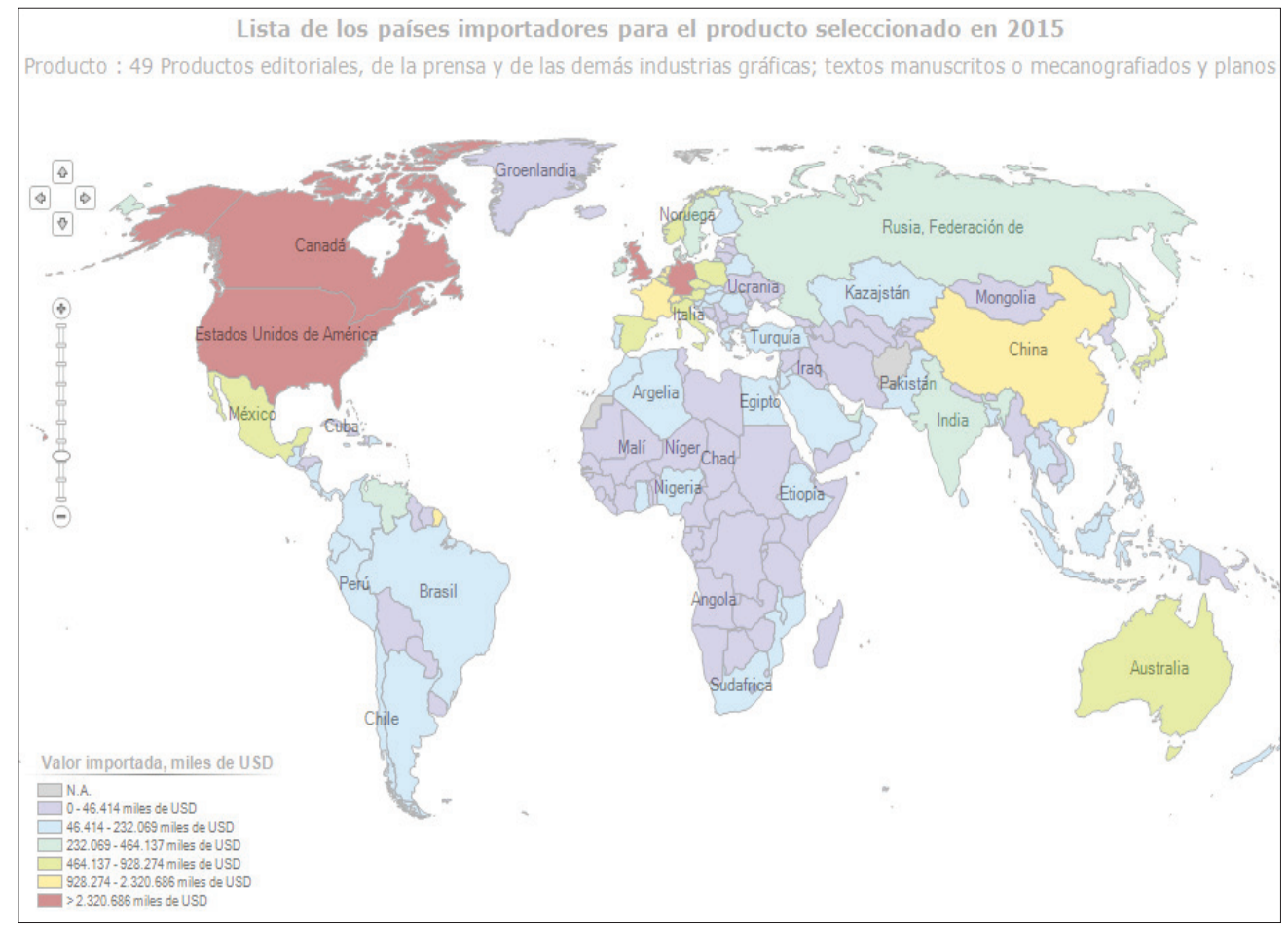

Fuente: Calculo de CCI basada en estadísticos de UM COMTRADE y Trade-Map (2015)

Una de las causas de la progresiva disminución de exportación de estos bienes es explicable a través del estudio de Silvano (2014), al plantear que uno de los problemas que debe enfrentar las exportaciones de libros impresos en Sudamérica es el idioma español. Para el autor, la falta de centros de producción en América Latina y la cláusula de exclusividad sobre el idioma español en los contratos firmados por editoriales españolas provocan que la mayor publicación de libros se realice en España y lleguen a América con el precio duplicado y prohibitivo. Adicionalmente, Silvano (2014) advierte que las editoriales de América Latina no están aprovechando el libro electrónico y la distribución en plataformas de acceso masivo. En las Tablas 3 y 4, se puede observar las exportaciones en miles de USD de Argentina y Chile de la partida 49 de los años 2012 y 2015, respectivamente. En ambos países, la exportación de libros, folletos, impresos, diarios y publicaciones periódicas ha bajado considerablemente en los últimos años. En Argentina, la disminución del monto exportado de la partida 49 es mayor a la observada en Chile que presenta una pequeña alza exportadora en álbumes y libros de estampas, calcomanías y sellos de correo, timbres fiscales y análogos destinados a tener curso legal.

Tabla 3. Exportación de Argentina Partida 49, Valores en Miles de USD (2012 y 2015)

\begin{tabular}{|l|r|r|}
\hline Descripción & 2012 & 2015 \\
\hline Libros, folletos e impresos similares, incluso en hojas sueltas. & 43.687 & 26.858 \\
\hline Diarios y publicaciones periódicas, impresos, incluso ilustrados. & 8.590 & 2.940 \\
\hline Álbumes o libros de estampas y cuadernos para dibujar o colorear, para niños. & 2.437 & 1.410 \\
\hline Música manuscrita o impresa, incluso con ilustraciones o encuadernada. & 109 & 26 \\
\hline Manufacturas cartográficas de todas clases. & 246 & 93 \\
\hline Planos y dibujos originales hechos a mano, de arquitectura, ingeniería, industriales, comerciales, topográficos o similares. & 199 & 12 \\
\hline $\begin{array}{l}\text { Sellos de correos, timbres fiscales y análogos, sin obliterar, que tengan o estén destinados a tener curso legal en el país en el que su valor } \\
\text { facial sea reconocido. }\end{array}$ & 148 & 32 \\
\hline Calcomanías de cualquier clase. & 482 & 209 \\
\hline Tarjetas postales impresas o ilustradas; tarjetas impresas con felicitaciones o comunicaciones personales, incluso con ilustraciones o adornos. & 263 & 95 \\
\hline Calendarios de cualquier clase impresos, incluidos los tacos de calendario. & 299 & 102 \\
\hline Los demás impresos, incluidas las estampas, grabados y fotografías. & 26.539 & 10.416 \\
\hline Total & 82.999 & 42.193 \\
\hline
\end{tabular}

Fuente: Elaboración propia, según datos ALADI (2017). 
Tabla 4. Exportación de Chile Partida 49, Valores en Miles de USD (2012 y 2015)

\begin{tabular}{|l|r|r|}
\hline Descripción & 2012 & 2015 \\
\hline Libros, folletos e impresos similares, incluso en hojas sueltas. & 15.510 & 4.922 \\
\hline Diarios y publicaciones periódicas, impresos, incluso ilustrados. & 1.616 & 884 \\
\hline Álbumes o libros de estampas y cuadernos para dibujar o colorear, para niños. & 457 & 804 \\
\hline Música manuscrita o impresa, incluso con ilustraciones o encuadernada. & 3 \\
\hline Manufacturas cartográficas de todas clases. & 2 \\
\hline Planos y dibujos originales hechos a mano, de arquitectura, ingeniería, industriales, comerciales, topográficos o similares. & 84 & 4 \\
\hline $\begin{array}{l}\text { Sellos de correos, timbres fiscales y análogos, sin obliterar, que tengan o estén destinados a tener curso legal en el país en el que su valor } \\
\text { facial sea reconocido. }\end{array}$ & 61 & 199 \\
\hline Calcomanías de cualquier clase. & 342 \\
\hline $\begin{array}{l}\text { Tarjetas postales impresas o ilustradas; tarjetas impresas con felicitaciones o comunicaciones personales, incluso con ilustraciones o } \\
\text { adornos. }\end{array}$ & 406 & 319 \\
\hline Calendarios de cualquier clase impresos, incluidos los tacos de calendario. & 144 & 144 \\
\hline Los demás impresos, incluidas las estampas, grabados y fotografías. & 19.253 \\
\hline Total & 26.890 \\
\hline
\end{tabular}

Fuente: Elaboración propia, según datos ALADI (2017).

\subsection{Comportamiento exportador}

Entre las variables que pueden estar influyendo en el comportamiento exportador de estos bienes creativos, se destacan:

a. Producto Interno Bruto (PIB) del País Importador y Exportador. Existe una correlación positiva entre los países con alto PIB per cápita de la última década (Estados Unidos; Canadá; Reino Unido; Francia; Islandia; Noruega; Suecia; Finlandia; Australia y Nueva Zelanda) y sus niveles de importación y exportación de bienes creativos en los últimos años.

b. Crisis Subprime: Domenech y Lazzoretti (2012) afirman que las industrias creativas de Sudamérica no han sido inmunes a los efectos de la crisis subprime.

c. Distancia y Costo de Exportación: Hoffman (2000) plantea que el comercio exterior de un país está estrechamente vinculado con su ubicación geográfica y así, los servicios de transporte que cubren las distancias hacia los mercados y los puertos son factores fundamentales para garantizar mayor eficiencia en el proceso exportador. La lejanía geográfica de Argentina y Chile con los principales importadores de bienes creativos puede estar obstaculizando la exportación de dichos bienes debido a los mayores costos de traslado. Adicionalmente, la salida a diferentes océanos de estos países, la infraestructura portuaria y su gestión logística son otros factores que pueden afectar los costos de exportación. Moreira et al (2013) consideran que tanto los responsables de la formulación de políticas como los investigadores Latinoamericanos a menudo pasan por alto la dimensión distributiva de los costos del comercio. En este estudio se concluye que la reducción de los costos internos de transporte permiten aprovechar al máximo las oportunidades de exportar. d. Facilitación comercial y emprendedora: En el estudio de Moïsé y Sorescu (2013) se concluye que la disponibilidad de información relacionada con el comercio, la simplificación y armonización de documentación y la automatización de procedimientos tienen mayor impacto en los volúmenes y los costos del comercio exterior de un país. Pinilla-Rojas (2016) concluye que para llevar a cabo un buen desarrollo de un proyecto creativo es necesario disponer de lineamientos legales y comerciales facilitadores, tales como permisos locativos, sistema de contratación y reglamentación legal.

\section{Estimación del modelo y metodología}

En esta investigación se utilizará un modelo gravitacional ampliado, mediante datos de panel estáticos con efectos fijos y aleatorios y dinámicos, con el propósito de determinar las variables que pueden estar influyendo en las exportaciones de objetos de arte, colección, antigüedad, editorial e industria gráfica de Chile y Argentina durante el período 2002-2015. El modelo gravitacional tiene como premisa el estudio efectuado por Newton (1687), sobre la Ley de Gravedad Universal que expresa que la fuerza ejercida entre dos cuerpos con sus respectivas masas y separados a una distancia, es directamente proporcional al producto de sus masas e inversamente proporcional a la distancia que los separa. El economista pionero en adaptar estos principios físicos al comercio exterior, fue Tinbergen (1962), donde en este caso, la atracción se mide por el flujo del comercio bilateral del país exportador a un país importador, siendo la masa de los cuerpos sustituida por el tamaño de la economías (usualmente medido por el PIB o producto nacional bruto) y la distancia como proxy de los costos de transporte. Teóricamente, a mayor tamaño de las economías del país exportador e importador mayor atracción gravitacional entre ellos, mientras que a mayor distancia geográfica menor atracción. 
Posteriormente, los autores que siguieron utilizando el modelo gravitacional para analizar el comercio internacional fueron Pöyhönen (1963) y Linnemann (1966), siendo Anderson (1979), Bergstrand (1985) y Helpman y Krugman (1985) los que le dieron sustento teórico. En las últimas décadas, el modelo gravitacional es uno de los modelos que más se utiliza para analizar los flujos de comercio internacional, migración o inversión extranjera debido a sus propiedades, soporte teórico y empírico, flexibilidad y adaptación a diferentes realidades regionales o de un país en particular. Además, se adapta a la estimación mediante datos de paneles estáticos y dinámicos.

Tradicionalmente, los métodos estadísticos que intentan explicar un fenómeno observado a través de una serie de variables han sido tratados mediante regresiones lineales, usando el método de Mínimos Cuadrados Ordinarios (MCO). En este estudio, el modelo lineal gravitacional ampliado estará dado por la ecuación expresada en logaritmos naturales (Ecuación 1) y estimada mediante MCO en datos de panel:

$\ln \left(E_{i j t}\right)=\beta_{0}+\mu_{1} \ln y_{i t}+\mu_{2} \ln y_{j t}+\xi_{1} \ln I F N E_{i t}+\xi_{2} \ln I F D L E_{i j}+\alpha_{1} \ln D_{i j}+\eta_{1} \ln \operatorname{Cose}_{i t}+\sum_{i} \delta_{k i} P_{i j}+u_{i j t}$

(Ecuación 1)

Donde ${ }^{1}$ :

i, j y t, representan al país exportador, país importador y año, respectivamente.

$E_{i j} \quad$ : Flujo de exportación del país $i$ al $j$.

$Y_{i, j} \quad$ : Producto Nacional Bruto Real del país $i$ y $j$ respectivamente.

$I F N E_{i} \quad$ : Índice de Facilidad para hacer negocios del país exportador (cercano a 1 significa reglamentación más favorable).

IFDLE $\quad$ : Índice de Fortaleza de los derechos legales del país exportador (0 es débil y 12 es fuerte)

$D_{i j} \quad$ : Distancia geográfica entre capitales de los países $i$ y $j$ en Kilómetros.

Cose $_{i} \quad$ : Costo de exportación por contenedor en USD.

Además, en la ecuación 1 se incluyen variables ficticias indicadas como $\left(\sum_{\mathrm{h}} \delta^{\mathrm{h}} \mathrm{P}_{\mathrm{ij}}\right)$. Así, el estimador MCO entregará separadamente los $\beta$ de estos efectos. Dichas variables ficticias asumirán valor 1 en caso de presentarse el evento o valor cero en caso de no presentarse el evento, las variables ficticias son:

$F_{i j} \quad:$ : Frontera común entre el país y el país.

$\mathrm{IC}_{\mathrm{ij}}^{i j} \quad$ : Idioma en común entre el país i y el país $\mathrm{j}$.

Mercosur $_{\mathrm{ij}}$ : Exportador e Importador miembros del Mercado Común del Sur (Argentina, Brasil, Paraguay, Uruguay y Venezuela desde 2014).
SA: $\quad$ : País exportador con salida al Océano Atlántico.

$\mathrm{SP}_{\mathrm{i}} \quad$ : País exportador con salida al Océano Pacífico.

Chile : : País exportador Chile.

Argentina : País exportador Argentina.

NAFTA-CL : Intercambio comercial entre países miembros del Tratado de Libre Comercio de América del Norte (NAFTA, Estados Unidos, Canadá y México) y Chile.

MERCOSUR-CL: Intercambio comercial entre países miembros del Mercado Común del Sur (Argentina, Brasil, Paraguay, Uruguay y Venezuela desde 2014) y Chile.

China-Cl : Intercambio comercial entre China y Chile.

UE-CL : Intercambio comercial entre países miembros de la Unión Europea y Chile.

2009 : variable tiempo que representa el periodo de crisis subprime.

La variable índice de facilidad para hacer negocios (IFNE) se incluye en la ecuación gravitacional (1) para determinar el impacto que puede tener la reglamentación y facilitación comercial en el volumen exportado de estos bienes creativos. Mientras más cercano a 1 mejor son las reglamentaciones para facilitar el comercio y la creación de nuevos negocios, destacándose a Dinamarca con 3; Hong Kong con 5 y Reino Unido con 6. Chile presenta un indicador de 48 y Argentina de 117 en el 2014 (Banco Mundial, 2016), observándose que en ambos países la reglamentación vigente dificulta la creación de nuevos negocios y la comercialización.

Además, se incluye en la Ecuación 1 el índice de fortaleza de los derechos legales y medidas regulatorias (IFDLE) con el propósito de evidenciar algún efecto en el comportamiento exportador de estos bienes creativos, principalmente con el derecho de autor y propiedad privada. Este índice cuando está más cercano a cero son más débiles los derechos legales y medidas regulatorias, mientras que, más cercano a 12 son más fuertes. Para el 2014, el promedio mundial de este indicador es 5,1, destacándose Nueva Zelanda con 12 y Australia y Camboya con 11, mientras que Yemen, Timor, Eritrea y Bolivia son los países que presentan un indicador igual a cero. Argentina y Chile se encuentran por debajo del promedio mundial con 2 y 4 , respectivamente (Banco Mundial, 2016).

El costo de exportación por contenedor ha ido aumentando progresivamente en el mundo de 1213,66 USD en el 2005 a 1559,8 USD en el 2014. Por ello, esta variable de costo se constituye en un importante y estratégico elemento de estudio, dado que puede estar perturbando negativamente el proceso exportador al afectar el precio competitivo de mercado y los tiempos de entrega. En el Gráfico 1, se puede observar que Argentina presenta costos de exportación mayores que el promedio mundial (1770 USD, 2014), mientras que Chile menores (910 USD, 2014).

(1) Las bases de datos que se utilizarán son: bases de datos de Trade-Map y Aduana de Argentina y Chile, montos exportados en millones de dólares FOB; www.wcrlars. usda,gov/cec/java/lat-long.htm, distancia en kilómetros entre capitales de los países en estudio; Banco Mundial, datos estadísticos en línea (http://datos.bancomundial.org/). 
Las variables ficticias incluidas en la Ecuación 1 permiten medir ciertos aspectos cualitativos, como son: a) Los acuerdos comerciales firmados entre Argentina y Chile con sus principales socios comerciales. El estudio de Valenzuela-Klagges y Espinoza-Brito (2015) concluye que el comercio intrabloque (MERCOSUR, NAFTA, CAN) ha tenido un efecto positivo y consolidado en América sobre el flujo del comercio bilateral entre los países que se integran; b) Frontera en común que busca diferenciar los efectos fronterizos del comercio con países vecinos del resto del comercio bilateral argentino y chileno, siendo además, una variable que medirá el uso mayoritario del transporte terrestre con estos países; c) Idioma en común es una variable que frecuentemente se incluye en la estimación del modelo gravitacional, principalmente porque se ha observado que socios comerciales con el mismo idioma tienden a tener mayor flujo comercial entre ellos. Por ejemplo, el estudio de Benavides, Olascoaga y Cuello (2015) concluye que el idioma en común entre socios afecta positivamente en las exportaciones del sector textil-confección de Colombia; d) Se incluye, además, la variable ficticia de tiempo que permite capturar el efecto temporal del año 2009 y las variables que representan la salida a los diferentes océanos para medir el efecto geográfico.

Gráfico 1. Costos de Exportación de Contenedor en USD: 2005-2014.

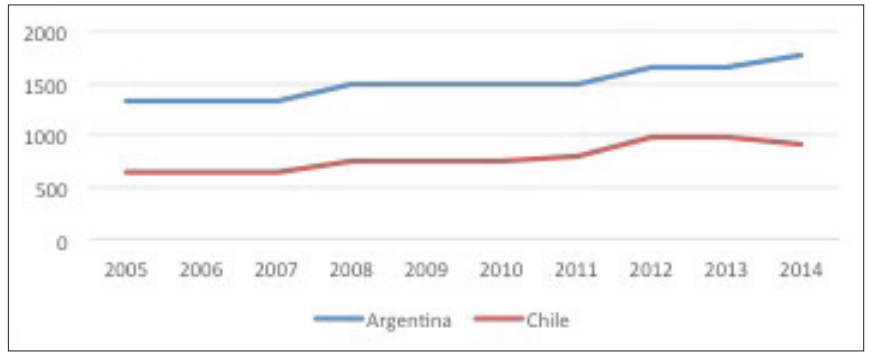

Fuente: Elaboración propia, según datos del Banco Mundial.

Para este estudio, se incluirán 37 países importadores, según zona geográfica, considerando los siguientes socios comerciales: 14 países pertenecientes a Europa; 1 a África; 4 a Asía; 2 a Medio Oriente; 3 a Norteamérica; 3 a América Central y el Caribe; 8 a Sudamérica y 2 a Oceanía. Dichos países constituyen el 96\% promedio de los destinos de exportación de estos bienes creativos de Argentina y Chile durante el período de estudio. La exclusión de algunos países se debe a la escasez o ausencia de intercambio durante uno o más años consecutivos en el período de estudio. El total de observaciones por año son 72 , constituyéndose un total de 1008 observaciones.

En relación a las observaciones cero, Santo-Silva y Tenreyro (2006) exponen un problema relacionado con la analogía entre la gravedad newtoniana y el comercio. $\mathrm{Al}$ respecto, señalan que la fuerza gravitacional puede ser muy pequeña, pero nunca es cero, mientras que, el comercio entre varios pares de países puede alcanzar valores cero, siendo un problema adicional el uso de logaritmos en la forma lineal de la ecuación gravitacional. Frente a esto y los posibles métodos de enfrentamiento a este problema, los autores plantean que sumarle $1 \mathrm{a}$ la variable dependiente o usar estimador tobit puede generar estimadores inconsistentes. En este estudio, se siguió una de las recomendaciones expuestas por Santo-Silva y Tenreyro (2006), optando por dejar una muestra de 37 países en que el flujo de comercio bilateral con Chile y Argentina no presentaba observaciones cero para el período en estudio, alcanzándose el 96\% de las exportaciones totales de obras de arte, colección, antigüedad, editorial e industria gráfica. Para determinar los montos totales de exportación, se utilizó los datos suministrados por la base de información Trade-Map y se complementó con datos e información entregada por las Aduanas de Argentina y Chile que especificaron, en algunos casos, los montos registrados menores a mil dólares.

Además, en esta investigación se cumple con la sugerencia de Roodman (2009), al usar una base de dato con un número de individuos mayores que 100 y por un período de tiempo (t) no mayor a 15 años.

La Ecuación 1 se estimará mediante datos de panel estático con efectos aleatorios, siendo el intercepto de la regresión aleatorio $\left(\beta_{0}\right)$ y que se considera como $\beta_{0}=\beta+u_{i}$. Es decir, en vez de considerar a $\beta$ como un intercepto fijo, se proyecta como una variable aleatoria con un valor medio $\beta$ y una desviación aleatoria $u_{i}$ de este valor medio.

La Ecuación 1 es adaptada para la estimación con datos de panel estáticos con efectos fijos (Ecuación 2). En la Ecuación 2 se excluyen todas las variables fijas que se mantienen a través del período en estudio, lo que permite observar intersecciones para todos los pares de socios comerciales, descartando una constante en común.

$\ln \left(E_{i j t}\right)=\mathrm{b}_{j}+\mathrm{m} \mathrm{h} y_{i}+\mathrm{m}_{2} \mathrm{~h} y_{j}+\mathrm{x}_{1} \mathrm{~h} I F N E_{i}+\mathrm{x}_{2} \mathrm{~h} I F D L E_{i}+\mathrm{h}_{1} \mathrm{~h} \operatorname{Cose}_{t}+u_{i j t}$

(Ecuación 2)

Donde $\beta_{\mathrm{ij}}$ es un número fijo para cada par de socios comerciales.

Adicionalmente a las estimaciones estáticas en datos de panel y dado lo expuesto por Baldwin y Venables (1995) y BID-Intal (2000), es conveniente adaptar y estimar la Ecuación 1 mediante datos de panel dinámicos. Baldwin y Venables (1995) plantean que los beneficios estáticos de procesos de integración y apertura comercial pueden ser cuantitativamente menores que los devengados de beneficios dinámicos, como los derivados de efectos escala y de producción. BID-Intal (2000) exponen que el comercio permite un avance en la especialización según las ventajas comparativas y aprovechamiento de economías de escala, disminuyendo gastos en actividades improductivas y mejorando continuamente la eficiencia de las empresas exportadoras hacia "best practices", evidenciando positivamente estos efectos en el comportamiento exportador de Colombia.

Por otra parte, Arellano y Bover (1990) exponen que uno de los aspectos en que el uso de panel resulta positivo y decisivo respecto a una muestra de corte transversal, es la posibilidad de modelizar respuestas dinámicas con microdatos. “...Ecuaciones con retardos de variables endógenas y exógenas pueden ser especificadas permitiendo la posibilidad de explicar procesos de ajustes" (pp. 5).

Dada esta posible endogeneidad del fenómeno exportador y su dinamismo, se recomienda incluir la estimación de datos de panel con 
efectos dinámicos y verificar el efecto y su significatividad del retardo de la variable dependiente. La ecuación 1 es adaptada para la estimación con datos de panel dinámicos (Ecuación 3).

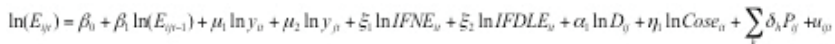

(Ecuación 3)

Siendo, $\mathrm{E}_{\mathrm{ijt}-1}$ el primer retardo de la variable dependiente. La Ecuación 3 se estimará mediante el estimador de Arellano y Bond (1991) y Blunder y Bond (1998) conocido como System GMM ya que utiliza las diferencias de los retardos, conformando un sistema de ecuaciones.

\section{Resultados}

En la Tabla 5 se puede observar los resultados con datos de panel estáticos con efectos aleatorios y fijos (Ecuación 1 y 2, respectivamente) y dinámico (Ecuación 3) para toda la muestra, corregida la heterocedasticidad. Con el propósito de comparar las diferencias entre el coeficiente de efectos fijos y aleatorio, se aplica la prueba de Hausman, obteniendo Prob $>\mathrm{Chi} 2=-5.4$. Al ser negativo el test y siguiendo las instrucciones de Motero (2005), se lleva este valor negativo a valor cero de la prueba de Hausman, por lo que se valida el efecto fijo. Por consiguiente, se ratifica que el método de efectos fijos es más conveniente que el efecto aleatorio para explicar el comportamiento exportador chileno y argentino en objetos de arte, colección, antigüedades, editorial e industria gráfica hacia los países en estudio. Sin embargo, el coeficiente de determinación alcanzado en la estimación mediante efectos aleatorios (0.11) es muy bajo. No se observa autocorrelación de variables, dado los resultados de autocorrelación de Wooldridge. Debido al bajo coeficiente de determinación, y que el test de Durbin Wu Hausman, detecta endogeneidad, se recomienda utilizar datos de panel dinámico (Ecuación 3).

Tabla 5. Resultados Estimación Ecuación 1, 2 y 3, Muestra Completa.

\begin{tabular}{|c|c|c|c|}
\hline Variables & $\begin{array}{l}\text { Efectos Fijos } \\
\text { Ecuación } 1\end{array}$ & $\begin{array}{c}\text { Efectos Aleatorios } \\
\text { Ecuación } 2\end{array}$ & $\begin{array}{l}\text { Efectos Dinámicos } \\
\text { Arellano-Blunder } \\
\text { Ecuación } 3\end{array}$ \\
\hline $\operatorname{Ln}(\operatorname{Exp}-1)$ & & & $0.26^{* * *}(0.04)$ \\
\hline Ln (PNB Exportador) & $2.2^{* * *}(0.49)$ & $2.59^{* * *}(0.45)$ & \\
\hline Ln (PNB Importador) & $1.59^{* *}(0.70)$ & $1.4^{* * *}(0.3)$ & $4.5^{\star * *}(1.1)$ \\
\hline Ln (Costo de Exportación) & $-2.7^{\star * *}(0.62)$ & $-1.8^{* * *}(0.6)$ & $-2.4^{* * *}(0.8)$ \\
\hline $\operatorname{Ln}(I F N E)$ & & & $-8.9 *(4.6)$ \\
\hline $\operatorname{Ln}(I F D L E)$ & & $2.6^{* * *}(0.8)$ & \\
\hline Chile & & & $-22.8^{* * *}(6.8)$ \\
\hline UE-Chile & & & $52.1^{* * *}(12.2)$ \\
\hline$I D$ & & & $-20.9^{* * *}(7.4)$ \\
\hline$F C$ & & & $-12.2^{* * *}(6.1)$ \\
\hline$S P$ & & & $8.7^{* * *}(6.9)$ \\
\hline Crisis & & & $-0.2 *(0.8)$ \\
\hline Constante & $30.6(29.2)$ & $-21.2^{\star \star *}(4.8)$ & $268.2^{* * *}(64.9)$ \\
\hline$R^{2}$ & 0.11 & 0.38 & \\
\hline Número Observaciones & 1008 & 1008 & 930 \\
\hline
\end{tabular}

Nota 1: Valores con heterocedasticidad corregida.

Nota 2: Nivel de significancia: ${ }^{* *}=0 \%$ error; ${ }^{* *}=0 \%<\mathrm{P} \leq 2.5 \%$; $^{*}=2.5 \%<\mathrm{P} \leq 5 \%$

Nota 3: Valores entre paréntesis es estándar de error.

En la Tabla 5, se observa los resultados de la estimación de la Ecuación 3 mediante datos de panel con efectos dinámicos con un retardo de la variable dependiente, utilizando la técnica de Arellano-Blundell. De acuerdo a los coeficientes estimados mediante datos de panel con efectos dinámicos (Ecuación 3, Tabla 5), es posible concluir que la endogeniedad disminuye y concuerda con lo teóricamente esperado. Los resultados indican que el primer retardo de la variable dependiente tiene signo positivo y significativo al igual que los coeficientes del producto nacional bruto real del importador y la salida al océano Pacífico de Chile. Los coeficientes de la distancia geográfica, 
costos de exportación por contenedor e indicador de facilitación para hacer negocios son negativos y significativos. El acuerdo UE-Chile afecta positivamente en el comercio bilateral chileno, mientras que el acuerdo Mercosur-Chile, China-Chile, Nafta-Chile presenta efectos negativos. El bloque regional MERCOSUR no presenta efectos significativos en el comercio de este tipo de bienes para Argentina al igual que la salida al océano Atlántico. Las variables ficticias Chile, idioma en común, frontera en común y crisis subprime presentan coeficientes negativos.

En la tabla 6, se puede observar los resultados por separado de Argentina y Chile al estimar la Ecuación 3 mediante datos de panel dinámico. Se observa entre países un efecto semejante a lo observado en la tabla 5, destacándose que en Argentina el efecto positivo del primer retardo de la variable dependiente el efecto es mayor que lo observado en Chile, mientras que los efectos negativos de distancia y costos de exportación por contenedor son menores en Chile; los coeficientes del indicador de facilitación para hacer negocios, idioma en común y frontera en común son negativos y significativos para los dos países, sin embargo, en el caso de Chile este efecto negativo es mayor.

Tabla 6. Resultados Estimación Ecuación 3 para Argentina y Chile.

\begin{tabular}{|l|l|l|}
\hline \multicolumn{1}{|c|}{ Variables } & \multicolumn{1}{|c|}{$\begin{array}{c}\text { ARGENTINA } \\
\text { Efectos Dinámicos } \\
\text { Ecuación 3 }\end{array}$} & $\begin{array}{c}\text { CHILE } \\
\text { Efectos Dinámicos } \\
\text { Ecuación 3 }\end{array}$ \\
\hline Ln (Exp-1) & $0.28^{* * *}(0.06)$ & $0.19^{* * *}(0.03)$ \\
\hline Ln (PNB Importador) & $4.4^{* * *}(1.0)$ & $4.7^{* * *}(1.2)$ \\
\hline Ln (Distancia Geográfica) & $-22.3^{* * *}(5.4)$ & $-15.3^{* * *}(4.8)$ \\
\hline Ln (Costo de Exportación) & $-2.8^{* * *}(0.9)$ & $-2.2^{* *}(0.8)$ \\
\hline Ln (IFNE) & $-5.5^{*}(2.6)$ & $-7.9^{*}(4.2)$ \\
\hline Mercosur-Chile & & $-20.5^{* * *}(6.9)$ \\
\hline NAFTA-Chile & & $-2.6^{* * *}(1.2)$ \\
\hline China-Chile & & $-2.7^{* *}(1.4)$ \\
\hline UE-Chile & & $52.1^{* * *}(12.2)$ \\
\hline ID & $-17.9^{* *}(5.4)$ & $-21.9^{*}(7.8)$ \\
\hline FC & $-8.2^{*}(6.1)$ & $-14.2^{*}(6.1)$ \\
\hline SP & & $8.7^{* *}(6.9)$ \\
\hline Crisis & $-0.2^{*}(0.8)$ & $-0.2^{*}(0.8)$ \\
\hline Constante & $180.2^{* *}(32.9)$ & $190.2^{* * *}(54.9)$ \\
\hline Numero Observaciones & & \\
\hline Ni: Vares con & 465 & \\
\hline
\end{tabular}

Nota 1: Valores con heterocedasticidad corregida.

Nota 2: Nivel de significancia: ${ }^{* *}=0 \%$ error; ${ }^{* *}=0 \%<\mathrm{P} \leq 2.5 \%$; ${ }^{*}$ $=2.5 \%<\mathrm{P} \leq 5 \%$

Nota 3: Valores entre paréntesis es estándar de error.

\section{Conclusiones y recomendaciones}

Debido al alza sostenible de la demanda mundial por bienes creativos, este mercado es una oportunidad para diversificar las exportaciones de Argentina y Chile y una estrategia económica para fomentar la creación de empleo, promover la cultura y economías creativas y el desarrollo de habilidades artísticas en la población, garantizando un crecimiento económico con inclusión. A pesar de su importancia, no se observa en Argentina y Chile una política cultural específica de apoyo directo a la exportación de bienes creativos y que potencie el desarrollo emprendedor en estos países, con facilitación comercial, asesoría de procesos exportables, información de ferias, convenios, subastas e instituciones internacionales especialistas en compra de estos bienes creativos.

Se observa que las exportaciones de Chile y Argentina en objetos de arte, colección, antigüedad, editorial e industria gráfica presentan una tendencia a disminuir su nivel de exportación y diversificación de destino, con un máximo de destino correspondiente a 37 países a través del tiempo, en contraposición con el aumento progresivo del mercado mundial en estos bienes creativos.

Los principales resultados indican que el comportamiento exportador de objetos de arte, colección, antigüedad, editorial e industria gráfica de estos países está explicado por un modelo dinámico, confirmando que existe un efecto positivo por el uso de mejores prácticas en el proceso de exportación de estos bienes, aprendidas en procesos anteriores. Probablemente, al romper el obstáculo fronterizo y formalizar contactos y redes internacionales, el artista o emprendedor artístico nacional logra desarrollar economías de escala dentro de un mercado de competencia imperfecta y ampliar su mercado externo. Además, se confirma que a mayor crecimiento económico del país importador y mejor facilidad reglamentaria y legal para hacer negocios en Chile y Argentina mayor será la exportación de estos bienes. Mientras que, a mayor costo de exportación por contenedor y distancia geográfica entre importador y exportador menor será el monto total exportado, afectando principalmente a Argentina, debido a que presenta elevados costos de exportación por contenedor. En este sentido, se sugiere para ambos países promover una legislación que facilite la creación de negocios, su comercialización y exportación, como también, mejorar la infraestructura portuaria, viabilidad y conexión de transporte a puertos y aeropuertos y servicios logísticos con el propósito de disminuir los costos de exportación por contenedor. Esto es congruente con el estudio de Rodríguez (2013) que, refriéndose al libro chileno como producto de exportación, enfatiza que es fundamental enfrentar la logística y todos sus desafíos pendientes y mejorar la colaboración público-privada para facilitar la exportación del libro chileno.

En este estudio se concluye que los destinos de exportación de estos bienes no se centran en países fronterizos ni en países con idioma español y se evidencia una mayor factibilidad para exportar estos bienes a países no hispanoamericanos. Esto sugiere al gestor artístico la importancia de dominar un segundo idioma, como es el inglés que ha sido declarado idioma universal y es el idioma oficial para todas las plataformas digitales.

También, se sugiere prestar asesoría a pequeños emprendedores artísticos independientes o agrupaciones en relación a los procesos de exportación, derechos y licencias para facilitar procesos de exportación, puesto que aunque exista una legislación adecuada para la facilitación comercial, no siempre es de conocimiento y manejo práctico del artista, coleccionista, diseñador y escritor. Adicionalmente, se sugiere reforzar la conectividad a internet con capacitación para trabajar en 
redes de plataforma que promuevan internacionalmente estos bienes creativos. Como lo afirma Delgado-Cantú (2016) el acceso a internet, el uso generalizado de aparatos móviles y otros medios de plataformas digitales han contribuido al crecimiento de las industrias creativas, mejorando la comunicación y acortando los tiempos y distancias entre importador y exportador.

Las exportaciones chilenas de estos bienes creativos se benefician, además, por la salida al Océano Pacífico y por el acuerdo con la Unión Europea, aunque Chile presenta una evolución más débil e inconstante en este mercado que Argentina. Por otra parte, los acuerdos de Chile con China y países miembros del NAFTA afectan negativamente en el proceso exportador chileno de estos bienes creativos.

\section{Referencias}

ALADI (Asociación Latinoamericana de Integración, 2016-2017). Comercio exterior, Datos Estadísticos. (http://consultawebv2.aladi. org/sicoexV2/jsf/comercio_exterior_item_arancelario.seam)

Alonso, J. y Gallego, A. (2011) Primera Aproximación a la Caracterización y Medición de las Industrias Culturales en Cali. Estudios Gerenciales, 27(120), 13-40.

Anderson, J. (1979). A Theoretical Foundation for the Gravity Equation. American Economic Review, 69(1), 106-116.

Arellano, M., y Bover, O. (1990). La Econometría de Datos de Panel. Investigaciones Económicas (segunda época), 14(1), 3-45.

Arellano, M. y Bond, S. (1991). Some tests of specification for panel data: Monte Carlo evidence and an application to employment equations. The Review of Economic Studies, 58(2), 277-297.

Baldwin, R. y Venables, A. (1995). Regional economic integration. Handbook of international economics, 3, 1597-1644.

Banco Mundial (2016-2017). Datos estadísticos (http://datos.bancomundial.org/)

Barciela, C.; López, M. y Melgarejo J. (2012) Los bienes culturales y su aportación al desarrollo sostenible. Servicio de Publicaciones, Universitat d'Alacant / Universidad de Alicante.

Beltrán, L. R. (2013). Comunicación para el Desarrollo en Latinoamérica: Una evaluación sucinta al cabo de cuarenta años. IV Mesa Redonda sobre Comunicación y Desarrollo organizada por el Instituto para América Latina (IPAL) en Lima, Perú, entre el 23 y el 26 de febrero de 1993.

Benavides, E., Olascoaga, L., y Cuello, D. (2015). Comercio exportador sector textil-confecciones en Colombia y departamento del Atlántico: Una aproximación a los modelos gravitacionales. Revista Lebret (7), 79-100.

Bergstrand, J. (1985). The Gravity Equation in International Trade: Some Microeconomic Foundations and Empirical Evidence. The Review of Economics and Statistics 71, 143-153.
BID-INTAL (2000). "El Impacto Sectorial del Proceso de Integración Subregional en la Comunidad Andina: Sector Lácteo y Sector Textil” Institutos de Estudios Rurales, Pontificia Universidad Javeriana, IER; Instituto Venezolano de Investigación Científicas, IVIC; Fedesarrollo, Colombia; Universidad del Pacífico, Perú. BID-INTAL, Argentina.

Blundell, R. y Bond, S. (1998). Initial conditions and moment restrictions in dynamic panel data models. Journal of Econometrics, 87(1), 115-143.

CEPAL, N. (2014). Estudio Económico de América Latina y el Caribe 2014: desafíos para la sostenibilidad del crecimiento en un nuevo contexto externo. Santiago de Chile.

Delgado-Cantú, V. (2016), México Como Exportador De Creatividad, Cultura Y Tecnología: Factores De Éxito. Universidad Autónoma de Nueva León, Facultad Contraloría Publica y Administración y Vincula Tégica, EFAN. ISSN: 2448-5101, Año2 Nº1.

Domenech, R., y Lazzeretti, L. (2012). Las industrias creativas en España: una panorámica. Investigaciones regionales: Journal of Regional Research, (22), 181-206.

EY (2015) El Primer Mapa Mundial de las Industrias Culturales y Creativas, Tiempos de Cultura- World Creative, Bajo la supervisión de Marc Fhermitter; Bruno Perrin; Solenne Blanc, supervisión de Oxford Economics, Apoyo de CISAC-UNESCO.

Helpman, E. y Krugman, P. (1985). Market Structure and Foreign Trade. Increasing Returns, Imperfect Competition, and the International Economy. Cambridge, MA. MIT Press (1985-1996).

Hoffman, J. (2000). Tendencias en el transporte marítimo internacional y sus implicaciones para América Latina y el Caribe. Ponencia presentada en la Reunión Anual de la AAPA, Veracruz.

Jarque, V. (2007). El tiempo detenido. Descubrir el Arte, (97).

Linnemann, H. (1966). An Econometric Study of International Trade Flows. Amsterdam: North Holland publishing Co.

Moïsé, E. y Sorescu, S. (2013). Trade Facilitation Indicators: The Potential Impact of Trade Facilitation on Developing Countries. Trade (144). OECD Publishing.

Moreira, M.; Blyde, J.; Volpe, C. y Molina, D. (2013). Muy Lejos para Exportar: Los Costos Internos de Transporte y las Disparidades en las Exportaciones Regionales en América Latina y el Caribe. Banco Interamericano del Desarrollo (BID).

Motero, R (2005): Test de Hausman. Documentos de Trabajo en Economía Aplicada. Universidad de Granada. España.

Ocampo, J. A. (2014). La Arquitectura Monetaria Y Financiera Internacional A La Luz De La Crisis. América Latina: su Arquitectura Financiera, 16.

Pérez, L. y Sánchez, C. (2011). Mercado del arte e intermediarios: una perspectiva actual. Laboratorio de Arte, (23), 537-550. 
Pinilla-Rojas, J. (2016). Estrategias gerenciales de innovación para atraer inversión extranjera en la industria del cine colombiano. Bachelor's thesis, Facultad de Ciencias Económicas, Universidad Militar Nueva Granada.

Pöyhönen, (1963). A Tentative Model for the Volumen of Trade between Countries. Weltwirtschaftliches Archiv 90, 93-99.

Rodríguez, R. (2013). El libro chileno como producto de exportación: Una experiencia en curso. Comunicación y Medios, (27), Pág-184.

Romero, A. (2016). Desempeño reciente y lecciones no aprendidas: las perspectivas económicas para América Latina y el Caribe/Recent Performance and Un-Learned Lessons: Economic Perspectives for Latin America and the Caribbean. Revista Economía y Desarrollo, 156(1).

Roodman, D. (2009). A note on the theme of too many instruments. Oxford Bulletin of Economics and Statistics, 71(1), 135-158.

Rowan, J. (2014). La cultura como problema: Ni Arnold ni Florida. Reflexiones acerca del devenir de las políticas culturales tras la crisis. Observatorio Cultural. Recuperado el, 12.

Santos-Silva y Silvana-Tenreyro (2006). The Log Of Gravity. The Review of Economics and Statistics, 88(4), 641-658

Silvano, A. (2014) Boletín Cultural y Bibliográfico, 48 (86) (Ejemplar dedicado a: Algunas miradas al libro y a la edición en Colombia), 29-39
Tinbergen, J. (1962). Shaping the World Economy. Suggestions for an International Economic Policy. New York: Twentieth Cetury Fund.

Trade-Map (2015). Estadísticas del comercio para el desarrollo internacional de las empresas. http://www.trademap.org/ visitada el $22 / 11 / 2016$.

UNESCO (2016) Área de Acción: Oficina de la UNESCO en Santiago. Oficina Regional de la UNESCO para América Latina y el Caribe. http://www.unesco.org/new/es/santiago/culture/creative-industries/

Urrea, M. y Guzmán, W. (2012).El galerismo y su relación con el mercado del arte en Chile. Tesis Proyecto Final para optar al Magister en Gestión Cultural. Profesor Guía: María Inés Silva. Universidad de Chile. Escuela de Postgrado. Disponible en http://www.repositorio.uchile.cl/handle/2250/112179

Valenzuela-Klagges, B. y Espinoza Brito, L. (2015). Regionalismo latinoamericano y comercio bilateral. Revista Pilquen, 18(2), 1-11.

Van der Pol, H. (2007) "Key Role of Cultural and Creative Industries in the Economy”, estudio del Instituto de Estadística de la UNESCO (Montreal)

Wto (2011) El comercio Mundial 2010, Organización WTO. https://www.wto.org/spanish/res_s/booksp_s/anrep_s/wtr11-1_s.pdf 